\title{
Characterizations of the Shakil-Kibria-Singh Distribution
}

\author{
G. G. Hamedani \\ Department of Mathematics, Statistics and Computer Science, \\ Marquette University, Milwaukee, USA
}

\begin{abstract}
Characterizations of a continuous univariate distribution due to Shakil, Kibria and Singh (2010) (SKS), based on a simple relationship between two truncated moments is presented. We also point out that some special cases of the SKS distribution can be characterized based on the hazard function.

Zusammenfassung: Charakterisierungen einer stetigen univariate Verteilung in Shakil, Kibria and Singh (2010) (SKS) werden präsentiert, welche auf auf einer einfachen Beziehung zwischen zwei gestutzten Momenten beruhen. Wir machen auch darauf aufmerksam, dass einige Spezialfälle der SKS Verteilung basierend auf die Hazardfunktion charakterisiert werden können.
\end{abstract}

Keywords: Characterizations, Truncated Moments, Hazard Function.

\section{Introduction}

The problem of characterizing a distribution is an important problem which has recently attracted the attention of many researchers. Thus, various characterizations have been established in many different directions. The present work deals with the characterizations of a continuous univariate distribution, due to Shakil, Kibria, and Singh (2010) (SKS), based on a simple relationship between two truncated moments. The probability density function (pdf) introduced by SKS is a solution of what is recently called generalized Pearson differential equation. We would first like to say a few words about the Pearson system and then take up the concept of the generalized Pearson system and of course the generalized Pearson differential equation generating this system.

Various systems of distributions have been constructed to provide approximations to wide variety of distributions (see, e.g., Johnson and Kotz, 1970). These systems are designed with the requirements of ease of computation and feasibility of algebraic manipulation. To meet the requirements, there must be as few parameters as possible in defining a member of the system. One of these systems is the Pearson system. A continuous distribution belongs to this system if its pdf $f$ satisfies a differential equation of the form

$$
\frac{1}{f(x)} \frac{d f(x)}{d x}=-\frac{x+a}{b x^{2}+c x+d}
$$

where $a, b, c$, and $d$ are real parameters such that $f$ is a pdf. The shape of the pdf depends on the values of these parameters. Pearson (1895) classified the different shapes into a number of Types I-VII. Many well-known distributions are special cases of Pearson Type distributions. 
Recently, some researchers have considered a generalization of (1) given by

$$
\frac{1}{f(x)} \frac{d f(x)}{d x}=\frac{\sum_{j=0}^{m} a_{j} x^{j}}{\sum_{j=0}^{n} b_{j} x^{j}},
$$

where $m, n \in \mathbb{N} /\{0\}$ and the coefficients $a_{j}$ and $b_{j}$ are real parameters. The system of continuous univariate pdf's generated by (2) is called a generalized Pearson system, which includes a vast majority of continuous pdf's.

Shakil et al. (2010) consider equation (2) when $m=2 p, n=p+1, a_{j}=0, j=$ $1, \ldots, p-1, p+1, \ldots, 2 p-1 ; b_{j}=0, j=0,1, \ldots, p, b_{p+1} \neq 0$ and $x>0$. The solution of this special case is the pdf $f$, given by

$$
f(x)=C x^{\nu-1} \exp \left(-\alpha x^{p}-\beta x^{-p}\right), \quad x>0, \quad \alpha \geq 0, \beta \geq 0, \quad \nu \in \mathbb{R},
$$

where $\alpha=-\frac{a_{2 p}}{p b_{p+1}}, \beta=\frac{a_{0}}{p b_{p+1}}, \nu=\frac{a_{p}+b_{p+1}}{b_{p+1}}, b_{p+1} \neq 0, p \in \mathbb{N} /\{0\}, C$ is the normalizing constant and termed as SKS distribution henceforth. Shakil et al. (2010) classified their newly proposed family into the following three classes:

Class I $\alpha>0, \beta=0, \nu>0$ and $p \in \mathbb{N} /\{0\}$. For this case the normalizing constant is $C=\frac{p \alpha^{\nu / p}}{\Gamma(\nu / p)}$.

Class II $\alpha=0, \beta>0, \nu<0$ and $p \in \mathbb{N} /\{0\}$. For this case the normalizing constant is $C=\frac{p}{\beta^{\nu / p} \Gamma(-\nu / p)}$.

Class III $\alpha>0, \beta>0, \nu \in \mathbb{R}$ and $p \in \mathbb{N} /\{0\}$. For this case the normalizing constant is $C=\frac{p}{2}\left(\frac{\alpha}{\beta}\right)^{\nu / 2 p} \frac{1}{K_{\nu / p}(2 \sqrt{\alpha \beta})}$, where $K_{\nu / p}(2 \sqrt{\alpha \beta})$ denotes the modified Bessel function of the third kind.

As a motivation, Shakil et al. (2010) provided the statistical applications of their results to a problem of forestry. They stated that "It is found that our newly proposed model fits better than gamma, log-normal and inverse Gaussian distributions. Since many researchers have studied the use of the generalized inverse Gaussian distribution in the fields of biomedicine, demography, environmental and ecological sciences, finance, lifetime data, reliability theory, traffic data, etc., we hope the findings of the paper will be useful for practitioners in various fields of theoretical and applied sciences." For a detailed treatment of these cases and their significance as well as related statistical analysis we refer the reader to Shakil et al. (2010).

\section{Characterization Results}

As we mentioned in the last paragraph of Section 1, the SKS distribution may have potential applications in many fields of studies. So, an investigator will be vitally interested 
to know if their model fits the requirements of SKS distribution. To this end, one will depend on characterizations of SKS distribution which provide conditions under which the underlying distribution is indeed a SKS distribution. In this section we present the characterizations of the SKS distribution with pdf (3) in terms of a simple relationship between two truncated moments. We like to mention here the work of Galambos and Kotz (1978), Kotz and Shanbhag (1980), Glänzel (1987, 1988, 1990), Glänzel, Telcs, and Schubert (1984); Glänzel and Win (1994), Glänzel and Hamedani (2001) and Hamedani (1993, 2002, 2006) in this direction. Our characterization results presented here will employ an interesting result due to Glänzel (1987), which is stated here (Theorem G below) for the sake of completeness.

Theorem $\mathbf{G}$ Let $(\Omega, \mathcal{F}, \mathbf{P})$ be a given probability space and let $H=[a, b]$ be an interval for some $a<b(a=-\infty, b=\infty$ might as well be allowed). Let $X: \Omega \rightarrow H$ be a continuous random variable with distribution function $F$ and let $g$ and $h$ be two real functions defined on $H$ such that

$$
\mathrm{E}[g(X) \mid X \geq x]=\mathrm{E}[h(X) \mid X \geq x] \lambda(x), \quad x \in H,
$$

is defined with some real function $\lambda$. Assume that $g, h \in C^{1}(H), \lambda \in C^{2}(H)$ and $F$ is a twice continuously differentiable and strictly monotone function on the set $H$. Finally, assume that the equation $h \lambda=g$ has no real solution in the interior of $H$. Then $F$ is uniquely determined by the functions $g, h$ and $\lambda$, particularly

$$
F(x)=\int_{a}^{x} C\left|\frac{\lambda^{\prime}(u)}{\lambda(u) h(u)-g(u)}\right| \exp (-s(u)) d u
$$

where the function $s$ is a solution of the differential equation $s^{\prime}=\frac{\lambda^{\prime} h}{\lambda h-g}$ and $C$ is a constant to make $\int_{H} d F=1$.

Remark 2.1 (a) In Theorem G, the interval $H$ need not be closed. (b) The goal is to have the function $\lambda$ as simple as possible. For a detailed discussion on the choice of $\lambda$, we refer the reader to Glänzel and Hamedani (2001) and Hamedani (1993, 2002, 2006).

The goal of this work is to characterize each of the three cases mentioned in the introduction, by employing Theorem G.

Case I $(\alpha>0, \beta=0, \nu>0$ and $p \in \mathbb{N} /\{0\})$. The pdf (3) will be of the form

$$
f(x)=C_{1} x^{\nu-1} \exp \left(-\alpha x^{p}\right) .
$$

This is a special case of the pdf of the generalized gamma distribution (see Appendix A for its pdf). The generalized gamma distribution (with $p \in \mathbb{R}^{+}$) has been characterized based on Theorem G in Hamedani (2006, Subsection 2.5). Certain special cases of the generalized gamma distribution are characterized based on the hazard function in Hamedani and Ahsanullah (2005, Subsection 2.10). 
Case II $(\alpha=0, \beta>0, \nu<0$ and $p \in \mathbb{N} /\{0\})$. The pdf (3) will be of the form

$$
f(x)=C_{2} x^{\nu-1} \exp \left(-\beta x^{-p}\right), \quad x>0, \quad \beta>0, \quad \nu<0, \quad p \in \mathbb{N} /\{0\} .
$$

This is a special case of the pdf of the Amoroso distribution (see Appendix A for its pdf). The Amoroso distribution (with $p \in \mathbb{R}^{+}$) has been characterized based on Theorem G in Hamedani (2011, Subsection 2.1). As explained in Hamedani (2011), no nontrivial characterizations based on hazard function is possible in this case.

Case III $(\alpha>0, \beta>0, \nu \in \mathbb{R}$ and $p \in \mathbb{N} /\{0\})$ We have the following characterization of the SKS distribution.

Proposition 2.2. Let $X: \Omega \rightarrow[0, \infty)$ be a continuous random variable and let $h(x)=$ $x^{p-\nu} \exp \left(\beta x^{-p}\right)$ for $x \in(0, \infty)$. The random variable $X$ has pdf (3) if and only if there exist functions $g$ and $\lambda$ defined in Theorem $\mathrm{G}$ satisfying the differential equation

$$
\frac{\lambda^{\prime}(x)}{\lambda(x) h(x)-g(x)}=\alpha p x^{\nu-1} \exp \left(-\beta x^{-p}\right), \quad x>0 .
$$

Proof. Let $X$ have pdf (3) and let

$$
g(x)=x^{p-\nu}\left(\alpha-\beta x^{-2 p}\right), \quad x>0
$$

and

$$
\lambda(x)=\alpha \exp \left(-\beta x^{-p}\right), \quad x>0 .
$$

Then

$$
\begin{gathered}
(1-F(x)) \mathrm{E}[h(X) \mid X \geq x]=\frac{C}{\alpha p} \exp \left(-\alpha x^{p}\right), \quad x>0, \\
(1-F(x)) \mathrm{E}[g(X) \mid X \geq x]=\frac{C}{p} \exp \left(-\alpha x^{p}-\beta x^{-p}\right), \quad x>0,
\end{gathered}
$$

and

$$
\lambda(x) h(x)-g(x)=\beta x^{-(p+\nu)}>0 \quad \text { for } x>0 .
$$

The differential equation (4) clearly holds.

Conversely, if $g$ and $\lambda$ satisfy the differential equation (4), then

$$
s^{\prime}(x)=\frac{\lambda^{\prime}(x) h(x)}{\lambda(x) h(x)-g(x)}=\alpha p x^{p-1}, \quad x>0,
$$

and hence

$$
s(x)=\alpha x^{p}, \quad x>0 .
$$

Now from Theorem G, $X$ has pdf (3). 
Corollary 2.3. Let $X: \Omega \rightarrow[0, \infty)$ be a continuous random variable and let $h(x)=$ $x^{p-\nu}\left(\alpha-\beta x^{-2 p}\right)$ and $g(x)=x^{p-\nu} \exp \left(\beta x^{-p}\right)$ for $x \in(0, \infty)$. The pdf of $X$ is (3) if and only if the function $\lambda$ has the form

$$
\lambda(x)=\frac{1}{\alpha} \exp \left(\beta x^{-p}\right), \quad x>0 .
$$

Remark 2.4. The general solution of the differential equation (4) is

$$
\lambda(x)=\exp \left(\alpha x^{p}\right)\left[-\int \alpha p x^{\nu-1} \exp \left(-\alpha x^{p}-\beta x^{-p}\right) g(x) d x+D\right], \quad x>0,
$$

where $D$ is a constant. One set of appropriate functions is given in Proposition 2.2.

The SKS pdf has unbounded support and one may be interested in a similar distribution but with bounded support. The following pdf is similar to that of a SKS pdf with bounded support and is a member of the generalized Pearson system.

$$
f(x)=C p x^{-(p+1)}\left(\beta-\alpha x^{2 p}\right) \exp \left(-\alpha x^{p}-\beta x^{-p}\right), \quad 0<x<\left(\frac{\beta}{\alpha}\right)^{\frac{1}{2 p}},
$$

where $\alpha>0, \beta>0$, and $p \in \mathbb{N} /\{0\}$ are parameters and $C=\exp \{2 \sqrt{\alpha \beta}\}$ is the normalizing constant.

The distribution function corresponding to the pdf in (5) is

$$
F(x)=C \exp \left(-\alpha x^{p}-\beta x^{-p}\right), \quad 0<x<\left(\frac{\beta}{\alpha}\right)^{\frac{1}{2 p}} .
$$

For the special case of $\alpha=\beta$, we have

$$
f(x)=\alpha p \exp (2 \alpha) x^{-(p+1)}\left(\beta-\alpha x^{2 p}\right) \exp \left(-\alpha\left(x^{p}-x^{-p}\right)\right), \quad 0<x<1,
$$

where $\alpha>0$ and $p \in \mathbb{N} /\{0\}$ are parameters.

It is easy to see that the pdf $f$ given by (5) satisfies (2)

$$
\frac{1}{f(x)} \frac{d f(x)}{d x}=\frac{\beta^{2} p-\beta(p+1) x^{p}-2 \alpha \beta p x^{2 p}-\alpha(p-1) x^{3 p}+\alpha^{2} p x^{4 p}}{\beta x^{p+1}-\alpha x^{3 p+1}} .
$$

Remark 2.5. Characterization results similar to Proposition 2.2 and Corollary 2.3 can be stated for the pdf in (5) with $p \in \mathbb{R}^{+}$as well.

\section{Appendix A}

The pdf of the Amoroso distribution is given by

$$
f(x ; a, \alpha, \tau, k)=\frac{1}{\Gamma(k)}\left|\frac{\tau}{\alpha}\right|\left(\frac{x-a}{\alpha}\right)^{\tau k-1} \exp \left\{-\left(\frac{x-a}{\alpha}\right)^{\tau}\right\},
$$

for $x, a, \alpha, \tau \in \mathbb{R}, k>0$, support $x \geq a$ if $\alpha>0, x \leq a$ if $\alpha<0$. 
We give below a table (Table 1) displaying four cases based on the signs of $\alpha$ and $\tau$ for the random variable $X \sim \operatorname{Amoroso}(a, \alpha, \tau, k)$. Without loss of generality we assume $a=0$.

Table 1

\begin{tabular}{c|cc} 
& $\tau>0$ & $\tau<0$ \\
\hline$\alpha>0$ & $X \sim \mathrm{GG}(\alpha, \tau, k)$ & $1 / X \sim \mathrm{GG}(1 / \alpha,-\tau, k)$ \\
$\alpha<0$ & $-X \sim \mathrm{GG}(-\alpha, \tau, k)$ & $-1 / X \sim \mathrm{GG}(-1 / \alpha,-\tau, k)$
\end{tabular}

For $\alpha>0$ and $\tau>0$, Amoroso $(0, \alpha, \tau, k)=\operatorname{GG}(\alpha, \tau, k)$, the generalized gamma distribution. The distributions of $-X$ (when $\alpha<0, \tau>0$ ), $1 / X$ (when $\alpha>0, \tau<0$ ) and $-1 / X$ (when $\alpha<0, \tau<0$ ) are all generalized gamma distributions.

\section{Acknowledgements}

The author is grateful to the referee for his suggestions improving the presentation of the work.

\section{References}

Galambos, J., and Kotz, S. (1978). Characterizations of Probability Distributions. A Unified Approach with Emphasis on Exponential and Related Models (No. 675). Berlin: Springer.

Glänzel, W. (1987). A characterization theorem based on truncated moments and its application to some distribution families (Vol. B). Dordrecht: Reidel.

Glänzel, W. (1988). A characterization of the normal distribution. Studia Scientiarum Mathematicarum Hungarica, 23, 89-91.

Glänzel, W. (1990). Some consequences of a characterization theorem based on truncated moments. Statistics, 21, 613-618.

Glänzel, W., and Hamedani, G. G. (2001). Characterizations of univariate continuous distributions. Studia Scientiarum Mathematicarum Hungarica, 37, 83-118.

Glänzel, W., Telcs, A., and Schubert, A. (1984). Characterization by truncated moments and its application to Pearson-type distributions. Zeitschrift für Wahrscheinlichkeitstheorie und Verwandte Gebiete, 66, 173-183.

Glänzel, W., and Win, I. (1994). A characterization tool for discrete distributions under Window(R). In R. Dutter and W. Grossmann (Eds.), COMPSTAT'94 (Vienna, 1994), Short Communications in Computational Statistics (p. 199-200).

Hamedani, G. G. (1993). Characterizations of Cauchy, normal and uniform distributions. Studia Scientiarum Mathematicarum Hungarica, 28, 243-247.

Hamedani, G. G. (2002). Characterizations of univariate continuous distributions II. Studia Scientiarum Mathematicarum Hungarica, 39, 407-424.

Hamedani, G. G. (2006). Characterizations of univariate continuous distributions III. Studia Scientiarum Mathematicarum Hungarica, 43, 361-385. 
Hamedani, G. G. (2011). Characterization of the Amoroso distribution. (Submitted) Hamedani, G. G., and Ahsanullah, M. (2005). Characterizations of univariate continuous distributions based on hazard function II. Journal of Statistical Theory and Applications, 4, 218-238.

Johnson, N. I., and Kotz, S. (1970). Distributions in Statistics. Continuous Univariate Distributions, Volumes 1 and 2. Boston, Mass.: Houghton Miffin Co.

Kotz, S., and Shanbhag, D. N. (1980). Some new approaches to probability distributions. Advances in Applied Probability, 12, 903-921.

Pearson, K. (1895). Contributions to the mathematical theory of evolution. Skew variation in homogeneous material. Philosophical Transactions of the Royal Society of London. A, 186, 343-414.

Shakil, M., Kibria, B. M., and Singh, J. N. (2010). A new family of distributions based on the generalized Pearson differential equation with some applications. Austrian Journal of Statistics, 39, 259-278.

Author's address:

G. G. Hamedani

Department of Mathematics, Statistics and Computer Science

Marquette University

Milwaukee, WI 53201-1881

U.S.A.

E-Mail: g.hamedani@mu.edu 